\title{
The Case of Blurring Memories: Reading Elizabeth is Missing
}

Shivangi Kanojia

M.Phil Research Scholar

Department of English

University of Delhi

New Delhi, India

shivangikanojia@yahoo.com

\begin{abstract}
This paper seeks to focus on the dimensions of a narrative about the narrator, the language employed. A narrative from the perspective of an older Dementia patient is studied, whose blurring memories are trying to explore the mysteries of her past and present. By doing this, the paper aims to investigate the constant cyclic shift in her narrative that changes the way meaning is looked upon, revises the metaphors attached to it. The paper also explores the story of aging and how aging is often seen from a negative viewpoint.
\end{abstract}

Keywords: Narrative Gerontology, Memory, Dementia, Aging, Social Construction.

Introduction

Human life is cyclic imbibing various dimensions, beginning from infancy and ending with old age. Old age is perceived as a phase where one gradually prepares oneself for the transition from human life. It is a natural cycle of the universe that ceases with the decline of the 
mortal human body. That is why a significant amount of importance is given to stories that convey a message and serve as a carrier of growth for others. Our lives are embedded within our personal stories that act as a gateway to a vast range of experiences, struggles, and adventures. Some of these stories are openly known to masses, while others are buried deep within the corners of our consciousness.

\section{Language and Cultural Significance}

In Narrative Gerontology in Research and Practice, Kate De Medeiros discusses narrative as a way of divulging some aspect of self with the intention of revelation (2). The purpose involves readers and listeners interpreting and applying the knowledge or experience derived, according to their understanding. This materializes a question that says why the narrator chooses to narrate a particular story. This is entirely dependent upon the narrator's choice, and this choice is a reflection of the narrator that gives its viewers a foreground to base their interpretation. The selection not only acts as a self-reflection but also operates as a window for the narrator to reflect and re-interpret part of self, revealed through his choice.

In itself, the language of the narrative adds another magnitude of complications to the structure of the narrative. In Narrative Gerontology in Research and Practice, Emery is quoted as describing "language as the system of codes and rules for the transmission of information" (De Medeiros, 3). This system involves a sound knowledge of the technical aspects of the language like grammatical structure, phonetic structure of sounds, lexicon, and semantics. This affects "the ability to encode and retrieve the symbols, signs, and sounds that comprise the language." (De Medeiros, 3) The language also includes "cultural symbols" shaped by culture or/and sub-culture, equally. Therefore, to form a semblance of a particular language style 
requires knowledge of its culture, expectations, and societal norms. Failure to comply leads to a distant interpretation of how the narrator intended the meaning to be while writing.

It is easy to limit oneself by equating the narrative with just a window that gives a glimpse into the narrator's world. As Medeiros reiterates in Narrative Gerontology in Research and Practice, a narrative is also grounded in a specific culture, history, text, context, and meaning. It is a vehicle of an expression embroiled in a particular culture that partakes within a particular type of storytelling, drawing on a unified cultural sense, reaching out to people with the same way of interpretation as carried out by the narrator. (De Medeiros, 10) Similarly, the narrative of aging gives insight into old age and makes the readers and viewers aware of the social binaries that construct the ideologies associated with growing old. Therefore, it reveals more about the construction of aging and paves the way towards a revelation of some aspect of the sense of self, which, in turn, interrogates the specific insight's social functioning.

Dementia and the breakdown of linear storytelling

Emma Healey's Elizabeth is Missing is not just a narrative of aging but an account of Maud's life story, who has Dementia. Maud is also the narrator in some parts of the description. Her tale is complicated by the condition that makes her lose hold over her present. That is why she is actively reminiscing about past events of her life, and steadily drawing parallels with her present situation. In a traditional narrative, there is a linear progression of events with a structure- beginning, middle, and end. Healey is flouting this structure by giving the reigns of the narrative to Maud, whose narration is always forgetting its purpose, i.e., to connect the narrative events to the central plot of the story. The problem with Maud's narrative is her constant insistence on going back to the past and due to her condition, a delay in connecting them to the 
present events. This delay works to the reader's advantage since the complete novel is about finding out the mystery behind Maud's sister, Sukey's disappearance, and Elizabeth's missing status. Healey employs another third-person narrator that recites Maud's past events with the clarity that Maud's narrative lacks. It helps in closing the gap between past and present, paving the way for readers to see Maud's narrative in a new light that magnifies the thread between Sukey and Elizabeth's disappearance.

Maud's story is a reflection of Maud's life-long desire to unravel the mystery surrounding her sister. That is why the snippets from her past focus on the events after her disappearance. Maud's nostalgic recollection is used in the broader narration to bring closure to the narrative. Each flashback holds the key to a clue that later, brought together by Maud with the help of third-person narrator and Helena, acts as a catalyst in bringing resolution. Also, the language given to Maud is symbolic of her gradually affecting condition. There is an absent narrator in Maud's narration style, almost as if a few sentences are given to justify her role. However, a great emphasis has been given to her internal monologues by the third-person narrator that reflects the workings of Maud's psyche. From here, readers understand Maud's helplessness in her inability to hold on to fading memories. Her need to be not treated like a child and be heard like a rational adult is continuously present in her monologues. The realization of her deteriorating condition overpowers her narration with the need to narrate whatever snippets of memory are left. Moreover, as the novel proceeds, the language in Maud's section also changes. Her internal monologue and conversation with others are mixed by chapter eighteen. There is a lack of differentiation that is seen in the absence of inverted commas. The deficiency of proper punctuation signifies Maud's rapidly deteriorating condition that makes her lose control over reality and suggests approaching cognitive disability 
In Narrative Gerontology in Research and Practice, Kate Medeiros quotes Jane Elliott's description of the critical features of narrative- chronology, meaning, and production for a particular audience (7). Elizabeth is Missing does not follow any element as the narrative continually goes back and forth in proceeding with the story. For instance, when the third person narrator talks about tracking Douglas's movements, the narrative structure changes back to the present, and we see Maud walking back from the library with Helena, her daughter. Her purposeful walk, when questioned by Helena by asking as to where she is going, she absently

Replies that she is following Douglas. As the narration progresses, Maud's past and present increasingly get blurred the point that we see her reaction to the flashbacks in her next present events. Nagging is experienced by not only Maud but by the readers, too. There is repeated reminder of forgotten details brought to the forefront by the past snippets of her memory, recollected through impactful events or memorable objects.

Another issue with her narrative is the constant shift in her thought process that adversely affects her mutterings' symbolic significance. Her story jumps from one thought to another without any completion. The shifting narrative leaves the structure in disarray wherein the readers felt a dire need for stable ground. Constant time travel results in an indefinite arrangement, but it also results in a continual change of meaning. Uncertain meaning and conclusion result from Maud's rapidly blurring memories that always seek solace in past events. Hence, persistent visiting and re-visiting adds new pieces, deletes some, and alters the remaining on every encounter. The constant alteration affects the already attached metaphors to that particular memory, and the meaning associated with it raises concerns over the present events as the past invades its paws onto one's immediate present. In doing so, it changes the current and 
our perception of the present, and sometimes, just like Elizabeth's son, it is not seen as an insightful experience but an unwanted intrusion.

Memory in Elizabeth is Missing is the field of contestation. Memory is the ability to stock and process information. When combined with the prowess of language, a person can project memory into an evolved human discourse. This, in turn, facilitates the process of comprehension, analysis, and further production. Memory is not a linear recording of the event with no bias and all the original details in place (Randall, 22). It is a process that is facilitated by a metaphor that invites us to look into our memories in a more meaningful way (Randall, 24). In the narrative of the aging, the constant evolution of meaning is necessary for experiencing overall development.

This meaning is given through the metaphoric association of memory (Randall, 24). Now, Healey presents us with a narrative that progresses with Maud's past events and her deteriorating memory. As she moves closer to unwiring the strings of her sister's murder mystery, she also unwires and loses bit by bit, her hold on her present. The basic structure of memory is overturned by Dementia wherein previously attached memory is lost, and a new one is too difficult to grasp. Even if grains of past events visit, as seen in Maud's case, memory gets blurred with the present facts, making a new deception of the mind. The meaning-making process that is implicit with the structure of memory gets obfuscated. However, forming meaning falls back to the friends, family, and caregivers of the Dementia patient. Helena acted as a catalyst in bringing closure to Maud's quest to find Suky's murderer. She served as the invisible thread articulating the distance between third-person narrator and Maud to not only readers but to Maud, herself.

Similarly, the narrative of aging is entwined within the structure of memory, metaphor, and meaning. The description renders the readers deep into the throes of life-long experiences, 
struggles, and, most importantly, a conscious outlook towards growing old. Each story of old age encounters its memory, maybe not in a linear fashion, but by adopting a style that brings the knowledge gained from many experiences to the forefront. The metaphor and meaning derived differs from individual to individual on the level of comprehension. The moment a type of comprehension is voiced, it acquires a definite structure just like any other narrative. However, it also opens avenues for a different awareness that will lead to a new meaning.

Maud and the perception of aging

Apart from being a Dementia patient, Maud is an older woman living in her house, often visited by her caregiver and daughter, Helena. Despite her condition, she frequently goes out and, when able to, asserts her independence. Emma Healey presents a narrative that slowly unleashes a hostile environment towards older people. She gives a glimpse into a culture that associates an item of specific clothing and conduct to growing old as evident in part wherein Maud comments on older people's necessity to carry reading glasses, false teeth, and a hearing aid to let the others know that they belong to a particular age group. This depicts society's need to form a commune that feels the need to allot characteristics to each age group. In Narrative Gerontology in Research and Practice, Kate Medeiros discusses social age-related to behavior deemed socially appropriate according to a person's actual age (19). This attributes to a negativistic impact on the way aging is viewed as about particular social order is akin to living in a restricted society. This brings forth the widespread discourse on aging that has its roots in the dogma of decay. The focus is not on the possible advancement of life but the images of decline, struggle, and end. Growing old is seen as an end to life and possible life adventures. A new type 
of restriction gets imposed that slowly limits one's physical as well as mental capabilities, and vulnerability becomes the looming shadow of older people's existence.

However, a new discourse has been initiated based on "successful aging," and opposes the unfavorable exchange on aging, as discussed by Sarah Lamb in Permanent Personhood or Meaningful Decline? Toward a critical anthropology of successful aging. (2) Lamb initiates discussion on the new positive aging that propagates independence and self-support. This attitude not only makes its older adults an active citizen but also paves the way towards reducing the burden on state and society. However, the positive discourse also has complication s within its structure. Its epicentre is in the anti-aging culture that contributes to community chasing the self, buried in their younger years. The obsession with looking younger procreated by the advent of anti-aging medical advancements further created a void within people's recesses that akin growing old to death. The toxic obsession perpetuates a culture that lives in constant denial of the cyclic human condition. In Hinduism, death and decay are a progressive cycle from birth to end, and a transition from human life. The cultural idea of aging perpetuates a different stance, which is concerned with accepting and embracing the changes accompanied by growing old. However, not everything needs to be viewed from the body-imaging aspect of healthy aging. The promotion of self-reliance emphasizes the ideals of a fit mind and body. This healthy lifestyle holds its daily activities in an active social life and productive life. As examined by Becca R. Levy, Martin D. Slade, and Stanislav V. Kasl in Longitudinal Benefit of Positive SelfPerceptions of Ageing on Functional Health, the positive viewing of the self affect one's conscious mental faculties and physical functioning to a great extent (1). This kind of selfprojection encourages a holistic mind and body and accepts the transience of human life. Also, this generates a positive outlook which in turn manufactures positive expectations of the self. 


\section{Conclusion}

The culture of constant labelling and categorization leads to negative imaging of self wherein there is continuous pressure to abide by certain norms, but it also hinders the individuality of a person. The culture of standard normative sets "ideal" standards for aging and divert to the set measure that excludes from the triumphant notion of aging. The media and socio-cultural discourse repeatedly reinforce the ideology that equates aging with the images of vulnerability, lack of beauty and vibrancy, frailty, and deterioration. Hence, we embody this perception, apply the same to self, and create false expectations that construct a harmful viewing of old age. The narrative of aging aims at getting a better understanding by studying the experiences, stories, and challenges of older people. The structure of the narrative is inter-related to the construction of memory, metaphor, and meaning. Maud's narrative structure creates complications within the structure of consciousness and meaning associated with it. It also explores the problem inherent within the socially constructed ideas of aging. 


\section{Works Cited}

De Medeiros, Kate. Narrative Gerontology in Research and Practice. New York: Springer Publishing Company, 2013.

Lamb, Sarah. "Permanent personhood or meaningful decline? Toward a critical anthropology of successful aging.” Journal of Aging Studies 29 (2014): 41-52.

Levy, Becca R, et al. "Longitudinal Benefit of Positive Self- Perceptions of Ageing on Functional Health.” Journal of Gerontology: Psychological Sciences 57B.5 (2002): 409417.

Healey, Emma. Elizabeth is Missing. London: Penguin Books, 2014.

Randall, William. "Memory, Metaphor, Meaning: Reading for Wisdom in the Stories of Our Lives," Storying Later Life: Issues, Investigations, and Interventions in Narrative Gerontology, ed. Gary M. Kenyon, Ernst Bohlmeijer; and William Lowell Randall, Cary: Oxford University Press, USA, 2010, pp. 20-38. 